To be published in Applied Optics:

Title: Image processing for safety assessment in Civil Engineering

Authors: Belen Ferrer, Juan Pomares, Ramon Irles, Julian Espinosa, and David Mas

Accepted: 21 May 2013

Posted: $\quad 24$ May 2013

Doc. ID: 185417 


\title{
Image processing for safety assessment in Civil Engineering
}

\author{
Belen Ferrer, ${ }^{1}$ Juan C. Pomares, ${ }^{1}$ Ramon Irles, ${ }^{1}$ Julian Espinosa, ${ }^{2}$ David Mas ${ }^{2,}{ }^{*}$ \\ ${ }^{1}$ Dept. of Civil Engineering, University of Alicante; P.O. Box, 99, O3080 Alicante (Spain) \\ ${ }^{2}$ Univ. Inst. Physics Applied to Sciences and Technologies, University of Alicante; P.O. Box, 99, O3080 Alicante (Spain) \\ *Corresponding author:david.mas@ua.es \\ Received Month X, XXXX; revised Month X, XXXX; accepted Month X, \\ XXXX; posted Month X, XXXX (Doc. ID XXXXX); published Month X, XXXX
}

\begin{abstract}
Behaviour analysis of construction safety systems is of fundamental importance to avoid accidental injuries. Traditionally, measurements of dynamic actions in Civil Engineering have been done through accelerometers, but high-speed cameras and image processing techniques can play an important role in this area. Here, we propose using morphological image filtering and Hough transform on high-speed video sequence as tools for dynamic measurements on that field. The presented method is applied to obtain the trajectory and acceleration of a cylindrical ballast falling from a building and trapped by a thread net. Results show that safety recommendations given in construction codes can be potentially dangerous for workers. (C) 2013 Optical Society of America

OCIS codes: 100.4999 Pattern recognition, target tracking, 120.4290 Nondestructive testing
\end{abstract}

\section{INTRODUCTION}

Traditionally, high-speed cameras in Civil and Mechanical Engineering have been used for visualization of fast phenomena and obtain qualitative data. Standard video cameras offered low time sampling frequencies that were not enough to register vibrations and fast movements. High speed cameras were expensive and the amount of produced data was difficult to analyse since large storage and computing capabilities were required. Because of this, other systems like accelerometers have been usually preferred. These devices are attached to the moving specimen and provide a single channel of data at very high sampling rates.

Despite their advantages, accelerometers are contact devices and their use is not always possible because of the inaccessibility of the measuring point. Additionally, they are very sensitive to high frequencies and information about lower frequency modes can be masked [1].

Reliable alternatives to accelerometers are Doppler interferometers that allow non-contact measurements. Nevertheless, these devices are very expensive and the distance between the target and the device must be short [2] so their use is not cost-effective for many applications.

In the last years, good quality high-speed cameras have been achievable at reasonable prices. In parallel, large and fast storage units and powerful computers are also becoming affordable. Therefore, image processing based methods become a reliable alternative to the above mentioned devices. In recent papers $[1,3]$, some of the authors show the possibility of using high-speed video sequences for measuring vibrations in structures.

Aside of vibrations, safety assessment in construction is an issue of obvious interest in Civil Engineering and it is regulated through different codes. We centre our interest on the edge protection in building constructions, and specifically on the study of the protective systems that prevent damage on workers accidentally falling to the void during the construction of sloped surfaces, which in Europe is regulated through the code EN13374 [4]. When trapping a falling body, these systems should be supple enough (a minimum deflection of $200 \mathrm{~mm}$ for class C) to avoid serious injuries to people. However, previous numerical studies [5] show that deflections of $200 \mathrm{~mm}$ may impose braking accelerations greater than those advisable for the human being $(8 g 10 g)[6]$.

The code establishes a safety-testing procedure consisting of a $75 \mathrm{~kg}$ cylindrical ballast falling from a $5 \mathrm{~m}$ height and a $60^{\circ}$ sloped working surface against the protection system (fig. 1), a surface made of a thread net tensed on a supporting structure [7]. Therefore, deflection of the system and acceleration of the ballast are the key point to determine whether the code which regulates the protective system is adequate and safe.

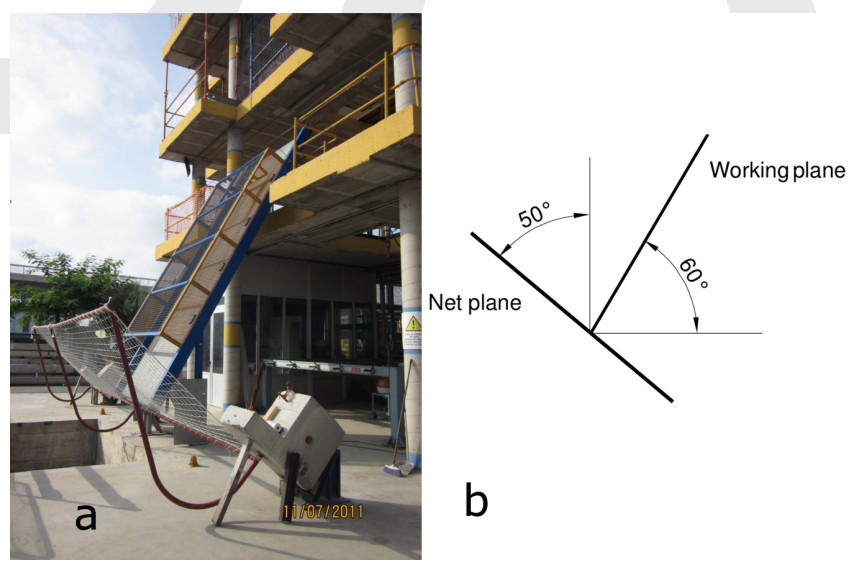

Fig. 1. (a) General view of the experiment. (b) Slopes of working surface and net.

The particular characteristics of the setup make difficult the use of measuring contact devices: the cylinder will rotate and fall onto a thread net. According to our previous experience, we propose using a high-speed camera to capture and analyse all the impact sequence.

The aim of this paper is to track the trajectory of the ballast and obtain the deflection of the protective system and the 
braking acceleration suffered by a falling body. As we said above use of high-speed image systems in Civil Engineering is not very common and, up to our knowledge, this work can contribute to expand the image processing techniques in that area.

The manuscript is structured as follows: first, in Section 2, we describe the experimental setup and the image processing algorithms to track the cylinder position. In Section 3, we describe the data processing and the main obtained results. Finally, the main conclusions of this work are outlined in Section 4.

\section{MATERIALS AND METHODS}

\subsection{Experimental setup}

The experimental setup was built according to the code EN13374 [4]: the working surface should be flat and have a slope of $60^{\circ}$. The human body is simulated with a cylinder body of $75 \mathrm{~kg}$, $300 \mathrm{~mm}$ of diameter and $1000 \mathrm{~mm}$ of length that is externally covered by a layer of rubber with a minimum thickness of $25 \mathrm{~mm}$ in order to have a soft surface. During the experiment, and previous to the free fall, the body centre-of-mass should cover a distance of $5 \mathrm{~m}$ on the ramp. The cylinder rolled down over the ramp until it reached the protection system. The setup was built in AIDICO facilities, in Valencia, Spain. In figure 2 (Media1), we show a video of the experiment where we can appreciate the speed of the process.

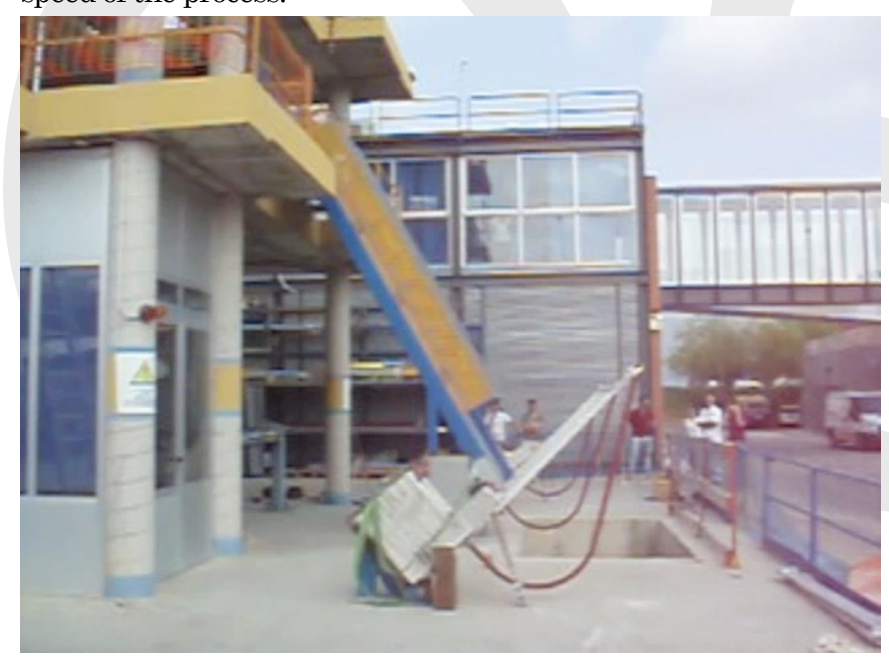

Fig. 2. (Media1) Video of the experiment at 30fps

As we pointed in the introduction, we are interested in measuring the deflection of the net and acceleration of the cylinder versus time. We monitored the experiments with an artificial vision system.

A video camera AOS X-Pri, working at 500 fps with a frame resolution of $800 \times 560 \mathrm{px}$ was used to capture the impact sequence. The camera was located in a lateral side of the net so that the expected impact point between the falling ballast and the net was in the centre of the field. This position also ensures that the axis of the cylinder is parallel to the optical axis of the camera (see figure 3), at least during the time span between the free fall and the rebound due to the net action.

The circular shape of the cylinder base is automatically detected in the scene. Tracking simple shapes in order to determine object movements in complex scenes is a common practice that simplifies very much the image processing algorithms [3]. As far as the cylinder axis is parallel to the optical axis of the camera, the obtained trajectory will be a good approximation of the cylinder trajectory. The instant position of the cylinder provides the trajectory and, through it, we obtain the maximum deflection of the protective system. Second derivative of trajectory equation allows us to calculate the acceleration suffered by the cylinder and, from that, infer possible damages to workers.

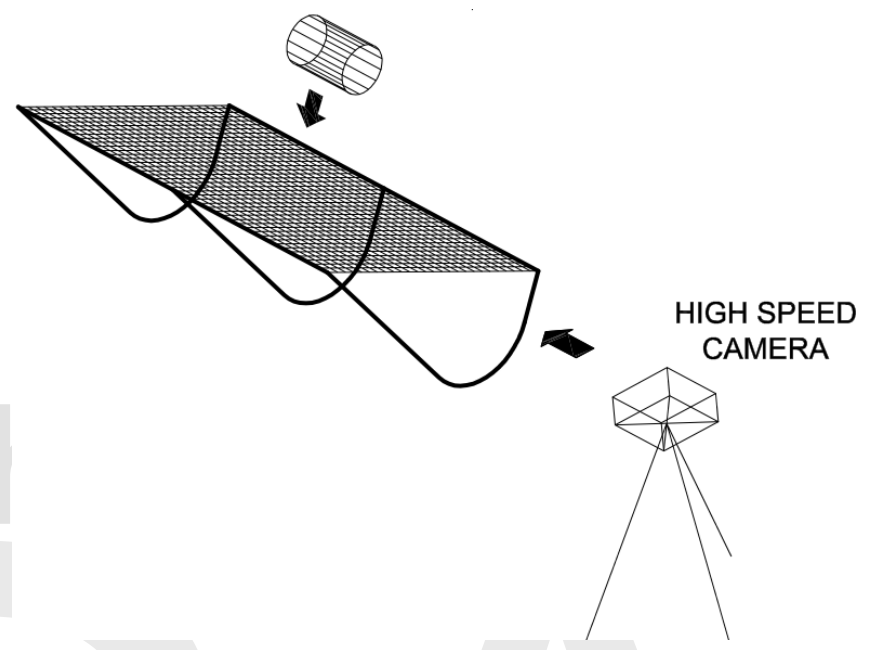

Fig. 3. Scheme of the setup with the high-speed camera location

Finally, notice that the camera provides object location in pixels. Real distances can be obtained just by comparing the diameter in pixels of the cylinder with its real size, thus giving a conversion parameter of $0.45 \mathrm{px} / \mathrm{mm}$. This parameter can be modified just by changing the lens magnification or the object-tocamera distance.

The acceleration of the cylinder was also obtained with an ICP tri-axial accelerometer, which is the common device used in Civil Engineering for these tasks. It was located on the basis of the hollow cylinder, thus having an eccentric position with respect to the rotation axis. The $\mathrm{x}$-axis was pointing towards the axis of the cylinder while the y-axis was tangent to the cylinder surface. Finally the z-axis registered the acceleration in the direction perpendicular to the trajectory plane. For the sake of clarity, we will rename the xyz-axes as normal, tangent and perpendicular axes, respectively.

\section{2.- Image processing algorithm}

Detection of the cylinder position consists of two basic steps. In the first step, the scene is thresholded and binarized and the borders extracted. Image cleaning is needed in order to remove as many lines as possible. In the second step, the presence of a circular shape is detected. In order to facilitate and accelerate the calculation, the position of the cylinder and its radius are manually estimated in the first frame. For the remaining frames of the sequence, the process is fully automatic.

Let us describe the process point by point. After the video capture, the sequence is moved from the camera to the computer and processed off-line with Matlab [8]. The first frame of the sequence is presented to the user and three points from the cylinder base (a circle) must be selected by hand. From these three points, we geometrically estimate the centre and the radius of the circle (see figure 4). 
Notice that the scene is relatively complex with many objects brighter than the cylinder itself. Thus, direct binarization will provide a noisy scene. However, major part of the scene remains static along the whole sequence so it does not add any important information. Therefore, we can take the absolute difference of two consecutive frames and then multiply this difference by the first one. With this, static parts are eliminated while moving parts are strongly enhanced. The image is then cleaned through a median filtering. A Sobel filter [9] extracts the borders of the image, thus providing a binary image. Additional cleaning of the image is done by using a $3 \times 3$ morphological opening filter that smoothes the lines and removes small white objects. Result of the process can be seen in figure 5 .

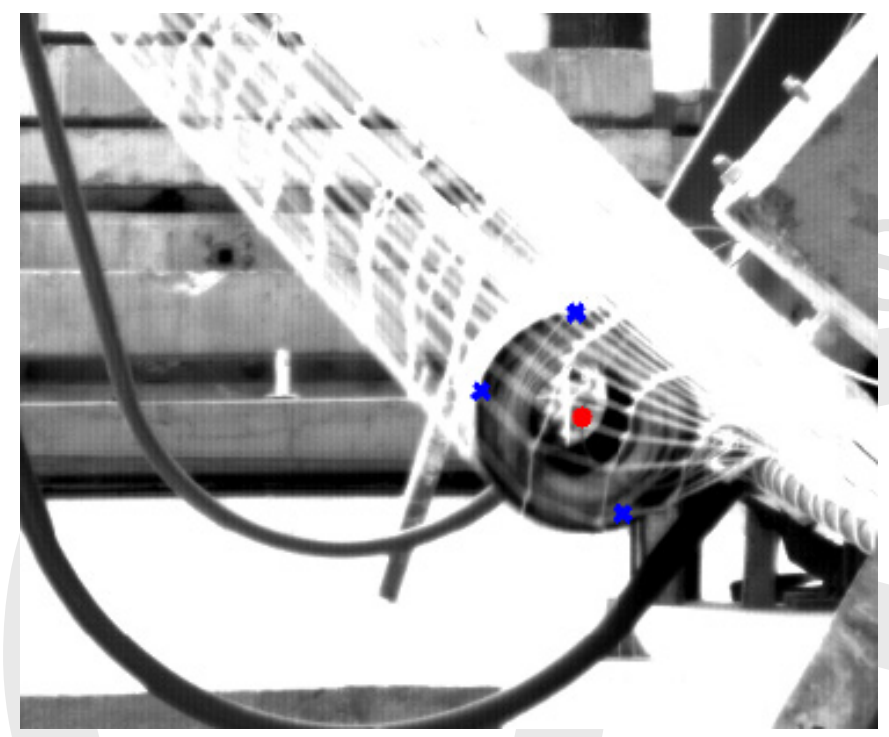

Fig. 4. Manual selection of three point of the circle (blue crosses) to obtain the centre of the circle (red dot)

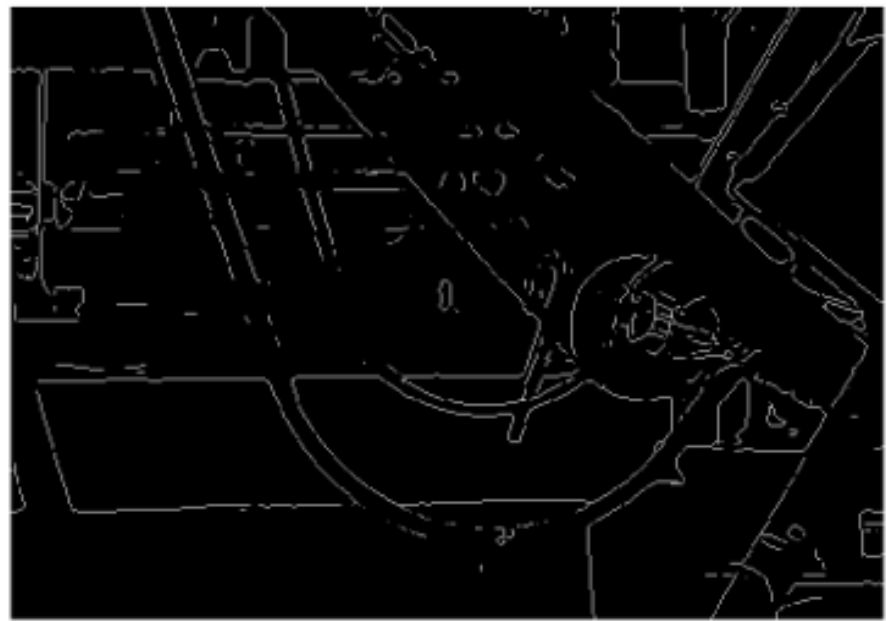

Fig. 5. Image after image processing method.

Even after severe noise removal, the image presents many lines that cross the cylinder basis profile. Moreover, the circular contour is uncompleted, with gaps that change with time. Therefore, simple tracking through simple image correlation did not provide reliable results. The Hough transform attempt to group edge points into object candidates by performing an explicit voting procedure over a set of parameterized object [10]. It is a technique used to detect features of a particular shape on a binary image. The process of identifying possible circular objects in Hough space is relatively simple. The algorithm traces circumferences of the desired radius that pass through a particular white pixel of the binary image and counts how many of the pixels in the image are likely to belong to any of these circumferences. Results of the pixel count are accumulated in one matrix and the exact position of the target is determined by the maximum of the accumulator matrix. The process is repeated for all white pixels in the scene.

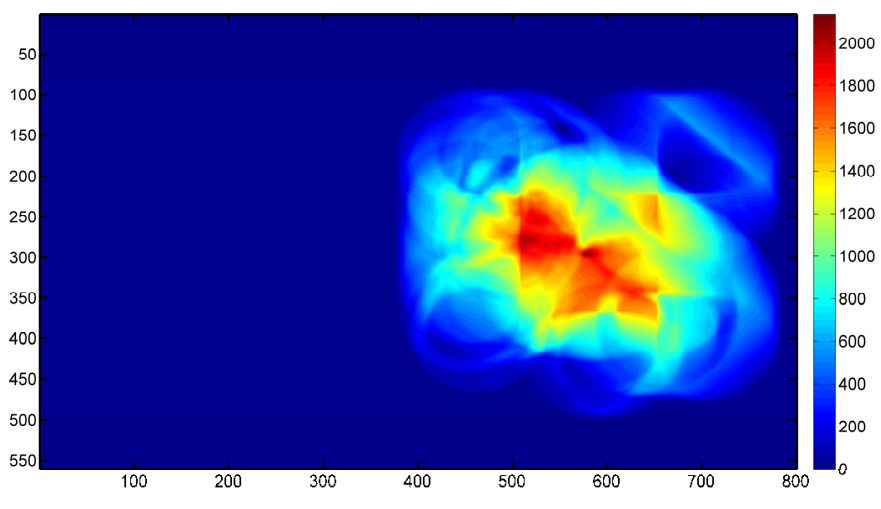

Fig. 6. Final result of the Hough transform, with the maximum locating the centre of the circumference. Units are in pixels and height units are arbitrary.

In our case, the radius of a circumference is given as an entrance parameter and the Hough transform will search for the exact position of the circumference centre. The centre estimated from the first frame will be used to define a region of interest, so the transform will be only applied on a local area of the image. In our case, the cylinder diameter is of $135 \mathrm{px}$, so the region of interest is defined as a squared window of $150 \times 150 \mathrm{px}$ centred in the estimated centre of the basis circumference.

The estimation of the radius may present some inaccuracies, due to cylinder oscillation or presence of noise. In order to maximize the detection possibilities, the algorithm is applied to an interval of radii of $\pm 3 \mathrm{px}$ around the expected value. Therefore, different accumulation matrixes are obtained and the absolute maximum from all of them is taken as the correct cylinder position. In figure 6 , we present the result of the accumulator for the scene in figure 5 .

After circumference detection, the obtained centre and radius are used as estimators to the following frame. Thus, from the first image, the algorithm runs automatically and detects the position of the cylinder at each frame. Figure 7 shows the result of the algorithm over a complete sequence. After that, the complete trajectory is obtained (Media2).

\section{RESULTS}

As we pointed before, the main results of the experiment are the maximum deflection of the net and the maximum acceleration suffered by the cylinder. All these results can be obtained from the cylinder trajectory in figure 7 with distances conveniently transformed into $\mathrm{mm}$, according to the previous conversion parameter.

First, we would like to underline that kinematic equations describe the movement of the cylinder centre-of-mass while, in our case, we observe the base of the cylinder. Thus, trajectory 
here obtained will coincide with the movement of the real body only while the axis of the cylinder is parallel to the camera axis. Once the cylinder rebounds in the net, the cylinder starts a complex movement and the alignment is lost, so the obtained trajectory does not have complete physical correspondence with the body movement. Nevertheless, we assume that during the measuring time, movement is relatively simple and the obtained trajectory corresponds with that of the centre-of-mass.

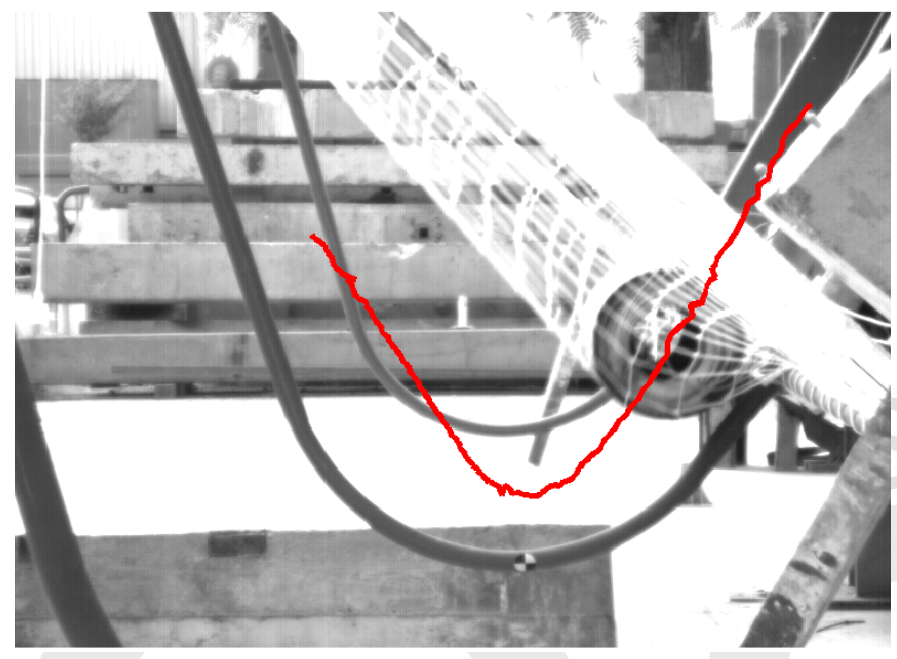

Fig. 7. Sequence of centre detection (Media2) and trajectory of the cylinder trapped by the net.

The maximum deflection of the system can be easily derived from the obtained trajectory: the initial point for this measure is the instant in which the ballast first touches the net and the final point is the farthest from the first one. In the experiment shown in Media2, the maximum deflection resulted of $700 \mathrm{~mm}$, which is much higher than the than the minimum stated by the code (200 $\mathrm{mm})[4]$.

Although the norm does not establish a limit, an important parameter for the proper design of security systems is the maximum acceleration suffered by the falling object when trapped by the net. This can be obtained by double-derivation of the trajectory equations. Unfortunately, inaccuracies in the image processing algorithm produce that the obtained trajectory is noisy and this impedes direct numerical derivative. Therefore, a local least-squares fitting of the trajectory to a polynomial equation was done. Since only the maximum acceleration is needed, the fitting was done around the peak value. Different degrees of the polynomial equation were used to assess the stability of the result [11]. The only boundary condition imposed on the fitting was to force a value between -4.5 and -5.5 for the second order coefficient so we assure that initial acceleration is around $-9.8 \mathrm{~m} / \mathrm{s}^{2}$. Once obtained the polynomial adjustment, second derivative of the fitting expression gives the cylinder acceleration. In figure 8, we show the acceleration curves obtained for the fittings of the trajectory to different polynomial orders. We do not have precise enough data for deciding which fitting is the most adequate. Nevertheless, we would like to underline that the key parameter here is the maximum value, which is stable all the three fittings considered.
Result from the camera show that the maximum acceleration suffered by the ballast is around $100 \mathrm{~m} / \mathrm{s}^{2}$ or, equivalently, around $10 \mathrm{~g}$. This value has been compared with the one obtained by the accelerometer. In figure 9 , we show the acceleration curves for the three axis of the accelerometer together with the one obtained through image processing.

As we said before, one of the accelerometer axes is parallel to the camera axis and, therefore perpendicular to the trajectory plane of the cylinder. The data obtained from this component are negligible, thus showing that the movement is produced mainly in one plane.

The other two axes are coplanar with the trajectory plane and they rotate with the cylinder, following a cycloid-like trajectory. The normal axis registers the centripetal acceleration, which is always aligned in the positive direction, while the tangential axis registers the acceleration due to changes in the rotation speed. Additionally, both axes also register the corresponding projection of the external forces acting on the centre-of-mass, which correspond to the gravity and to the net reaction. These accelerations determine the cylinder trajectory. Notice that all accelerations, i.e. centripetal $\mathrm{ac}_{\mathrm{c}}(\mathrm{t})$, tangential, $\mathrm{at}_{\mathrm{t}}(\mathrm{t})$ and due to external forces acting on the centre-of-mass, $\mathrm{acm}(\mathrm{t})$ depend on time.

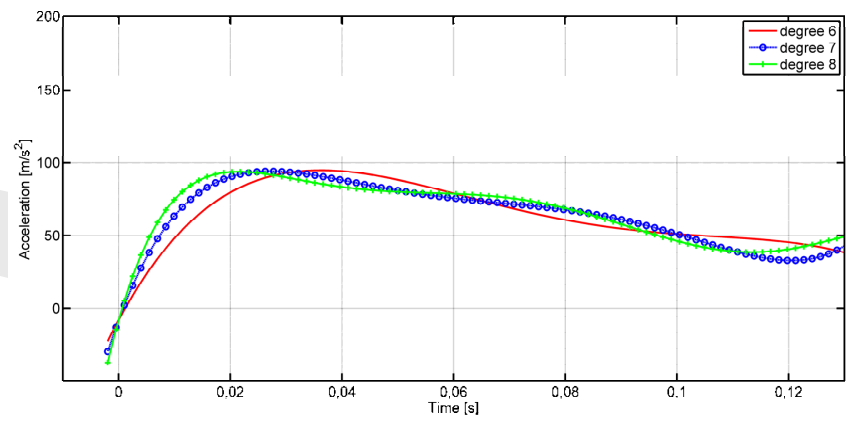

Fig. 8 Second derivative of the trajectory fittings to different polynomial degrees

Depending on the azimuthal position of the accelerometer, the external acceleration will add or subtract from those due to the cylinder rotation. Therefore, at one instant the normal axis will register accelerations in the interval $\left[\mathrm{a}_{\mathrm{c}}{ }^{-} \mathrm{a}_{\mathrm{cm}} ; \mathrm{a}_{\mathrm{c}}+\mathrm{a}_{\mathrm{cm}}\right]_{\mathrm{t}}$, while the tangential axis will register values in the range $\left[a_{t}{ }^{-} a_{c m} ; a_{t}+a_{c m}\right]_{t}$. From these data, an estimation of the peak acceleration suffered by the centre-of-mass should be obtained.

According to the position of the accelerometer on the cylinder basis and by analysing cylinder rotation in the video sequence, we obtain that the centripetal acceleration during the event between $50 \mathrm{~m} / \mathrm{s}^{2}$ and $70 \mathrm{~m} / \mathrm{s}^{2}$. Taking into account that the peak obtained by the accelerometer normal axis is around $140 \mathrm{~m} / \mathrm{s}^{2}$, we can estimate that the maximum acceleration due to external forces value should be between $70 \mathrm{~m} / \mathrm{s}^{2}$ and $90 \mathrm{~m} / \mathrm{s}^{2}$ or even higher. There is no evidence that both centripetal and external acceleration are exactly aligned at the moment when the last reaches its peak. Nevertheless, those moments must be close, so we consider that the interval obtained is a good estimation. 


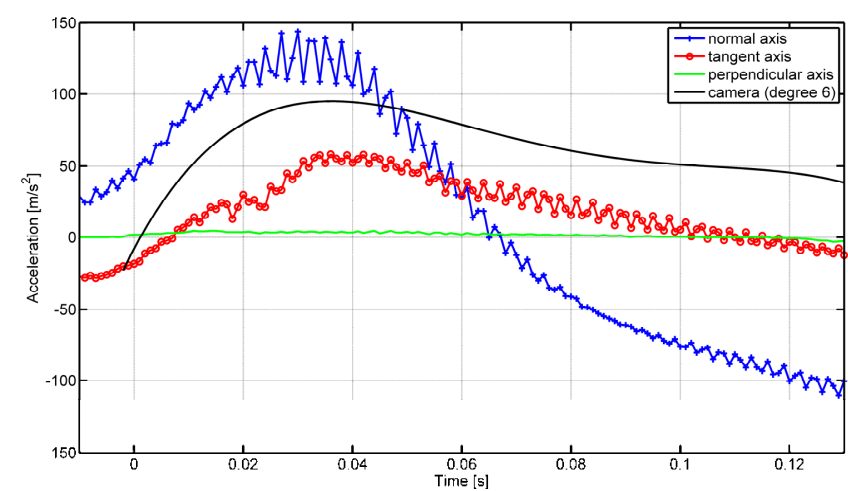

Fig. 9 Comparison results of acceleration obtained from accelerometer and form image processing methods (only the 6th degree polynomial fitting has been plotted).

Regarding the tangential acceleration, it mainly depends on the interaction between the cylinder and the net, which is difficult to analyse. Since the tangent and normal components are orthogonal, maxima of the curves will not coincide. Thus, it is expected that curves of both axes are out of phase and thus, may not register the highest peak. With the camera, we only registered the movement of the centre-of-mass and therefore, the values go between the maximum and minimum of the normal and tangential acceleration curves.

For security studies, the maximum peak value is always considered. The value here obtained $(\sim 14 \mathrm{~g})$ by the accelerometer is overestimated since it also considers rotational accelerations. Nevertheless, it is not a problem since overestimation of the acceleration maximum peak will lead to overprotective designs, thus resulting safe enough for human beings.

In order to confirm the results, a second experiment with the same thread net was performed. In this second trial, the knots of the thread were tighter and thus, it is expected to absorb less energy. Accordingly, the acceleration suffered by the ballast should be higher.

Values for this second experiment were $500 \mathrm{~mm}$ for the deflexion and $13 \mathrm{~g}$ for the acceleration measured through the camera, in front of a peak of $16 \mathrm{~g}$ obtained by the accelerometer.

With these results, the reader should notice that accelerations on the falling body obtained here are in the limit of the human body safety. We can infer that a system with a minimum 200 $\mathrm{mm}$ imposed by the code [4] can impose severe accelerations to the falling body and be potentially dangerous for a falling worker. Therefore, we can conclude that norms in the code are too lax and a revision of the norm is advisable.

\section{CONCLUSIONS}

In this paper we have described a non-invasive image-based method to perform a normalized experiment according to safety norms in building construction. With our proposal we determine the movement of a falling cylinder once it is trapped by the security net. The method uses a high-speed video camera working at $500 \mathrm{fps}$ to capture the movements. The video sequence is processed off-line with our own designed software, implemented in Matlab. The lateral base of the cylinder is recognized and tracked through the sequence by means of the Hough transform. Once started the procedure, the algorithm runs automatically and detects the position of the cylinder at each frame. Through the trajectory, maximum deflection of the protective system and maximum acceleration suffered by the ballast can be determined.

The acceleration of the cylinder was also measured with a triaxial accelerometer. Data from the accelerometer resulted to be of difficult interpretation: on the one hand, it registers together accelerations due to external forces and to the rotation of the cylinder. On the other hand, the axes of the accelerometer are intrinsic to the falling body and thus, register a changing projection of the external acceleration. Separation of all these effects in order to obtain the center-of-mass acceleration during the whole event requires the accelerometer instantaneous position which, in general, cannot be obtained through double integration [1] even with simpler movements.

Results obtained through optics and image processing are easier to interpret and, up to our understanding, more accurate. Additionally, image based method presents several advantages over traditional methods. Aside of simultaneous measurement of position and acceleration, our proposal allows direct visualization of the experiment and additional qualitative analysis (complex movement of the ballast, rebound time and trajectory, behaviour of the supporting structure) that have not been studied here. The method also is cost effective compared with other traditional systems. In this sense, it is even possible its implementation with low cost cameras [3] provided that pixel to millimetre conversion factor allows accurate enough determination of the trajectory.

Finally, the experiments here performed show that the norm [4] about edge protection systems in building construction may be too lax in their safety considerations and a revision should be considered.

\section{ACKOWLEDGMENTS}

The authors acknowledge the support of the Spanish Ministerio de Economía y Competitividad through the project BIA2011-22704 and the University of Alicante through the project GRE10-09. D. M. also acknowledges the support of the Generalitat Valenciana through the project PROMETEO/2011/021

\section{REFERENCES}

1. B. Ferrer, J. Espinosa, J. Perez, S. Ivorra, D. Mas, "Optical scanning for structural vibration measurement" Res. Nondestruct. Eval. 22, 61-75 (2011).

2. H.N. Nassif, M. Gindy, J. Davis, "Comparison of laser Doppler vibrometer with contact sensors for monitoring bridge deflection and vibration" NDT \& E Int. 38, 213-128 (2005)

3. D. Mas, J. Espinosa, A. B. Roig, B. Ferrer, J. Perez, C. Illueca, "Measurement of wide frequency range structural microvibrations with a pocket digital camera and sub-pixel techniques," Appl. Opt. 51, 2664-2671 (2012).

4. EN 13374 "Temporary edge protection systems - Product specification, test methods" European Commitee for Standardization (2004)

5. J.C. Pomares, E.G. Segovia, R. Irles "Personal protection rails for strong impacts." 4th Int. Confer. on Safety and Security Engineering, SAFE 2011. Antwerp (Belgium), 4-6 July 2011.

6. M. Voshell "High Acceleration and the Human Body" http://csel.eng.ohio-state.edu/voshell/gforce.pdf

7. R. Irles, J.C. Pomares, "Sistema de protección de bordes para barandillas tipo B y tipo C" Patent pending $\mathrm{n}^{\circ}$ P201130987 (2011) 
8. Matlab Image processing toolbox (Mathworks Inc.), http://www.mathworks.es/help/toolbox/images/

9. R.C. Gonzalez, R.E. Woods, S.L. Eddins "Digital Image Processing Using MATLAB" (Prentice-Hall, 2003)

10. L. Shapiro, G. Stockman. "Computer Vision" (PrenticeHall, 2001)

11. B. Ferrer, J.C. Pomares, R. Irles, J. Espinosa, D. Mas, "High speed image techniques for construction safety net monitoring in outdoor conditions", Proc. SPIE 8436 (2012).
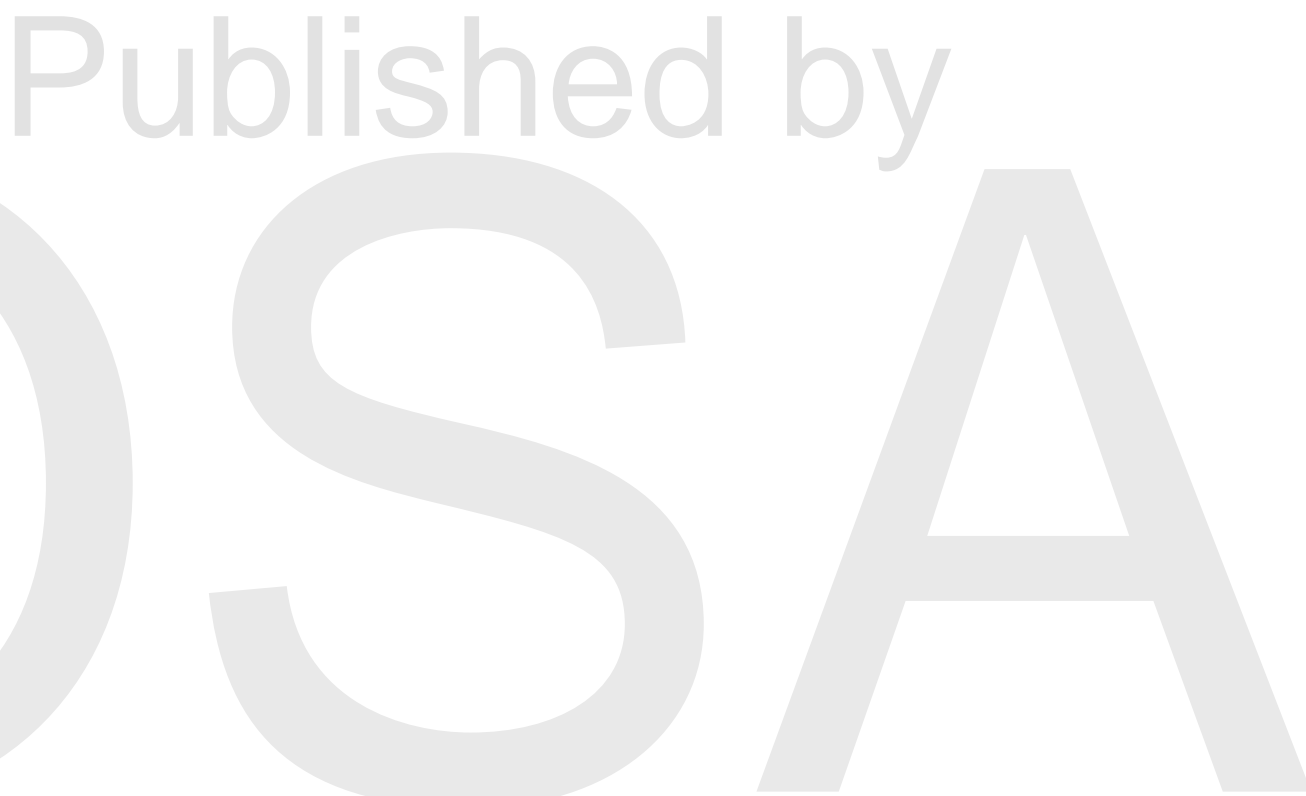